\title{
Phase Contrast Image Restoration via Dictionary Representation of Diffraction Patterns
}

\author{
Hang $\mathrm{Su}^{1,3}$, Zhaozheng $\mathrm{Yin}^{2}$, Takeo Kanade ${ }^{3}$, and Seungil Huh ${ }^{3}$ \\ 1 Department of EE, Shanghai Jiaotong University \\ 2 Department of CS, Missouri University of Science and Technology \\ 3 The Robotics Institute, Carnegie Mellon University*
}

\begin{abstract}
The restoration of microscopy images makes the segmentation and detection of cells easier and more reliable, which facilitates automated cell tracking and cell behavior analysis. In this paper, the authors analyze the image formation process of phase contrast images and propose an image restoration method based on the dictionary representation of diffraction patterns. By formulating and solving a min- $\ell_{1}$ optimization problem, each pixel is restored into a feature vector corresponding to the dictionary representation. Cells in the images are then segmented by the feature vector clustering. In addition to segmentation, since the feature vectors capture the information on the phase retardation caused by cells, they can be used for cell stage classification between intermitotic and mitotic/apoptotic stages. Experiments on three image sequences demonstrate that the dictionary-based restoration method can restore phase contrast images containing cells with different optical natures and provide promising results on cell stage classification.
\end{abstract}

\section{Introduction}

Computer-aided image analysis of phase contrast microscopy [1] has attracted increasing attention since it enables long-term monitoring of the proliferation and migration processes of live cells. Among the tasks of microscopy cell image analysis, cell detection and segmentation is one of the most fundamental components in that various analyses can be performed based on it. Cell detection and segmentation in phase contrast microscopy is challenged by clustered cells, cell shape deformation, and image artifacts such as bright halos and shade-off.

The common techniques employed for cell segmentation include thresholding [2], edge detection, and morphological operations [3]. These methods often fail when the contrast between cells and background is low. Another group of segmentation algorithms that are based on intensity gradient of images, namely, watershed [4], active contours [5], and level set [6], are sensitive to the initializations and local noisy gradients. To address these drawbacks, a restoration-based segmentation was recently proposed [7]. The method models the image formation process of phase contrast microscope to restore phase retardation caused by

\footnotetext{
* This research is supported by funds from Cell Image Analysis Consortium of Carnegie Mellon University and University of Missouri Research Board.
} 
cells, based on which cells are detected. However, this method fails to segment cells when they are bright in phase contrast microscopy images, e.g., mitotic or apoptotic cells, because the model assumes that the phase retardation caused by cells is small, which is not valid when cells become thick and thus appear bright in phase contrast microscopy.

In this paper, we revisit the phase contrast imaging model in [7], and propose a novel restoration algorithm based on dictionary representation of diffraction patterns, which can restore phase contrast images with various phase retardations. The proposed dictionary-based method restores a feature vector corresponding to diffraction patterns for each pixel. After the image restoration, high quality segmentation is achieved by clustering the feature vectors. Furthermore, since the restored feature vectors embed phase retardation information, cells can be classified between different stages, particularly between intermitosis and mitosis/apoptosis(dead).

\section{Methodology}

\subsection{The Image Formation of Phase Contrast Microscope}

The phase contrast microscope converts the phase difference in light passing through the transparent specimen to brightness changes in the image [1]. The wavefront of the illuminating beam is divided into two components after passing through the specimen. The primary component is the surround wave ( $S$ wave) that passes through or around the specimen without interacting with it. The other component is the diffracted wave ( $D$ wave) that is scattered by the specimen. These two waves undergo interference and produce a resultant particle wave ( $P$ wave). The cells can be observed only when the amplitudes of $P$ wave and $S$ wave are significantly different.

In [7, the surround wave $l_{S}$ and the diffracted wave $l_{D}$ are derived as:

$$
l_{S}=i \zeta_{p} A e^{i \beta}, \text { and } l_{D}=\zeta_{c} A e^{i(\beta+\theta(x))}+\left(i \zeta_{p}-1\right) \zeta_{c} A e^{i(\beta+\theta(x))} \cdot \operatorname{airy}(r),
$$

where $A$ and $\beta$ are the amplitude and phase of the incident light, respectively; $\zeta_{c}$ and $\zeta_{p}$ are the amplitude attenuation factors caused by cells and phase ring, respectively; $\theta(x)$ is the phase retardation caused by the specimen at location $x$; and $\operatorname{airy}(r)$ is an obscured Airy pattern (diffraction pattern with a bright region in the center surrounded by a series of concentric dark and bright rings [7]). The particle wave $l_{P}$ is calculated as $l_{P}=l_{S}+l_{D}$.

During the imaging model derivation in [7], the exponential terms in equation (1) are approximated using $e^{i \theta(x)} \approx 1+i \theta(x)$. Note that this approximation is valid only when the phase retardation $\theta(x)$ is close to zero. The assumption is apparently not applicable to general cases since $\theta(x)$, which is a function of the refractive indices and the thickness of cells, often vary along with different cell types and stages; more formally, the phase retardation $\theta$ can be calculated as:

$$
\theta=\frac{2 \pi}{\lambda}\left(n_{1}-n_{2}\right) t
$$


where $\lambda$ denotes the wavelength of the incident light; $t$ is the thickness of the cell; and $n_{1}$ and $n_{2}$ denote the refractive indices of the cell and medium, respectively.

\subsection{Dictionary Representation of Diffraction Patterns}

In this paper, we propose a generalized imaging model by approximating the term $e^{i \theta(x)}$ in Eq. (11) using a linear combination of $\left\{e^{i \theta_{m}}\right\}$ :

$$
e^{i \theta(x)} \approx \sum_{m=0}^{M-1} \psi_{m}(x) e^{i \theta_{m}} \text {, s.t. } \sum_{m=0}^{M-1} \psi_{m}(x)=1 \text { and } \psi_{m}(x) \geqslant 0 \text {. }
$$

where $\left\{\theta_{m}\right\}=\left\{0, \frac{2 \pi}{M}, \cdots, \frac{2 m \pi}{M}, \cdots, \frac{2(M-1) \pi}{M}\right\}$. We impose the nonnegative constraint because the solution would not be unique without it as $\psi_{m}(x) e^{i \theta_{m}}=$ $-\psi_{m}(x) e^{i\left(\theta_{m}+\pi\right)}$. Moreover, the nonnegative constraint removes the absolute operator of $\ell_{1}$ norm, allowing the problem to be solved in a standard manner.

The intensity of the observed image $g$ is calculated as:

$$
\begin{aligned}
g & =\left\|l_{p}\right\|^{2}=\left(l_{S}+l_{D}\right) \cdot\left(l_{S}+l_{D}\right)^{*} \\
& =A^{2}\left\{\begin{array}{l}
\zeta_{p}^{2}+\zeta_{c}^{2}-2 \zeta_{c}^{2} \cdot \operatorname{airy}(r)+\left(\zeta_{p}^{2}+1\right) \zeta_{c}^{2} \cdot(\operatorname{airy}(r))^{2}+ \\
\zeta_{p}^{2} \zeta_{c}\left(e^{-i \theta(x)}+e^{i \theta(x)}\right) \cdot \operatorname{airy}(r)+i \zeta_{p} \zeta_{c}\left(e^{-i \theta(x)}-e^{i \theta(x)}\right)
\end{array}\right\} .
\end{aligned}
$$

Substituting the exponential terms in Eq. (4) with Eq. (3) yields a linear representation of the observed image:

$$
\begin{aligned}
g & =A^{2}\left\{\begin{array}{l}
\zeta_{p}^{2}+\zeta_{c}^{2}-2 \zeta_{c}^{2} \cdot \operatorname{airy}(r)+\left(\zeta_{p}^{2}+1\right) \zeta_{c}^{2} \cdot(\operatorname{airy}(r))^{2}+ \\
\sum_{m=0}^{M-1} \psi_{m}(x)\left(\left(2 \zeta_{p} \zeta_{c} \sin \theta_{m} \cdot \delta(r)+2 \zeta_{p}^{2} \zeta_{c} \cos \theta_{m} \cdot \operatorname{airy}(r)\right)\right.
\end{array}\right\} . \\
& =C+D \sum_{m=0}^{M-1} \psi_{m}(x)\left(\sin \theta_{m} \cdot \delta(r)+\zeta_{p} \cos \theta_{m} \cdot \operatorname{airy}(r)\right)
\end{aligned}
$$

where $\delta(\cdot)$ is a Dirac delta function, $C$ is a constant that indicates the items unrelated to the feature vector $\psi_{m}(x)$, and $D$ is also a constant. $C$ can be eliminated by flat-field correction [1/7] and thus we ignore it for simplicity. Hence,

$$
\begin{aligned}
g & \propto \sum_{m=0}^{M-1} \psi_{m}(x)\left(\sin \theta_{m} \cdot \delta(r)+\zeta_{p} \cos \theta_{m} \cdot \operatorname{airy}(r)\right) \\
& \triangleq \sum_{m=0}^{M-1} \psi_{m}(x) \operatorname{PSF}\left(\theta_{m}\right),
\end{aligned}
$$

where $P S F$ denotes the point spread function; i.e., $P S F\left(\theta_{m}\right)$ represents the diffraction pattern with phase retardation $\theta_{m}$. In our experiments, $\zeta_{p}$ was set between 0.4 and 0.5 based on the information of microscope we used. 
We discretize $\operatorname{PSF}\left(\theta_{m}\right)$ into a $(2 T+1) \times(2 T+1)$ kernel, $(u, v)$ element of which is denoted by $\widehat{P S F}\left(\theta_{m}, u, v\right)$. Then, from Eq. (6), the imaging model of $(i, j)$ pixel of $g$ is also discretized as:

$$
g(i, j)=\sum_{m=0}^{M-1} \sum_{u=1}^{2 T+1} \sum_{v=1}^{2 T+1} \psi_{m}(i+u-T-1, j+v-T-1) \widehat{P S F}\left(\theta_{m}, u, v\right) .
$$

We define $\Psi_{m}$ as the vectorized representation of the matrix $\left\{\psi_{m}(i, j)\right\}$ and $h_{m}(i, j)$ as the vector obtained by vectorizing the sparse matrix whose $(i-T$ : $i+T, j-T: j+T)$ submatrix is $\left\{\widehat{P S F}\left(\theta_{m}, u, v\right)\right\}$ and the other elements are zero. Then, Eq. (7) is simplified into:

$$
g(i, j)=\sum_{m=0}^{M-1} h_{m}(i, j)^{T} \Psi_{m}
$$

and thus the vectorized form of the phase contrast microscopy image $\{g(i, j)\}$ can be modeled as:

$$
g=\sum_{m=0}^{M-1} H_{m} \Psi_{m}, \text { s.t. } \Psi_{m} \geqslant 0
$$

where $H_{m}$ is the matrix obtained by stacking up row vectors $\left\{h_{m}(i, j)^{T}\right\}$ in order. Note that when $g$ consists of $P$ pixels in total, $H_{m}$ is a $P \times P$ matrix, each row of which contains only $(2 T+1) \times(2 T+1)$ non-zero elements.

\subsection{The Restoration of the Phase Contrast Images}

Based on the theory of sparse representation [8], we formulate the following optimization problem to restore the feature vector from Eq. (9):

$$
\min \sum_{k=0}^{N-1}\left\{\left\|\Psi_{m_{k}}\right\|_{1}+w_{s} \Psi_{m_{k}}^{T} \mathbf{L} \Psi_{m_{k}}\right\} \text { s.t. }\left\{\begin{array}{l}
\left\|g-\sum_{k=0}^{N-1} H_{m_{k}} \Psi_{m_{k}}\right\|_{2}<\varepsilon \\
\Psi_{m_{k}} \geqslant 0
\end{array}\right.
$$

where $\mathbf{L}$ is a Laplacian matrix defining the similarity between spatial pixel neighbors [7; $w_{s}$ is the weight determining the spatial smoothness, which was set between 0.2 to 0.4 in our experiments; and, $N$ is the number of representative retardations, the optimal value of which can vary with the cell type and property.

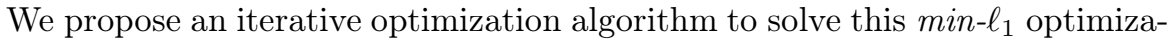
tion problem since it is known that there is no closed-form solution for such a problem. We first search the best-matching $N$ bases in the dictionary $\left\{H_{m}\right\}$ with the matching pursuit algorithm [8], and then utilize a non-negative multiplicative updating method [9] to obtain the nonnegative feature vectors $\left\{\Psi_{m_{k}}\right\}$. The procedure is described in Algorithm 1.

Solving Eq. (10) yields the best set of $\left\{\Psi_{m_{1}}, \cdots, \Psi_{m_{k}}\right\}$, which means that each pixel is restored as a feature vector. We apply $K$-means clustering on the feature vectors to segment cells in images. 


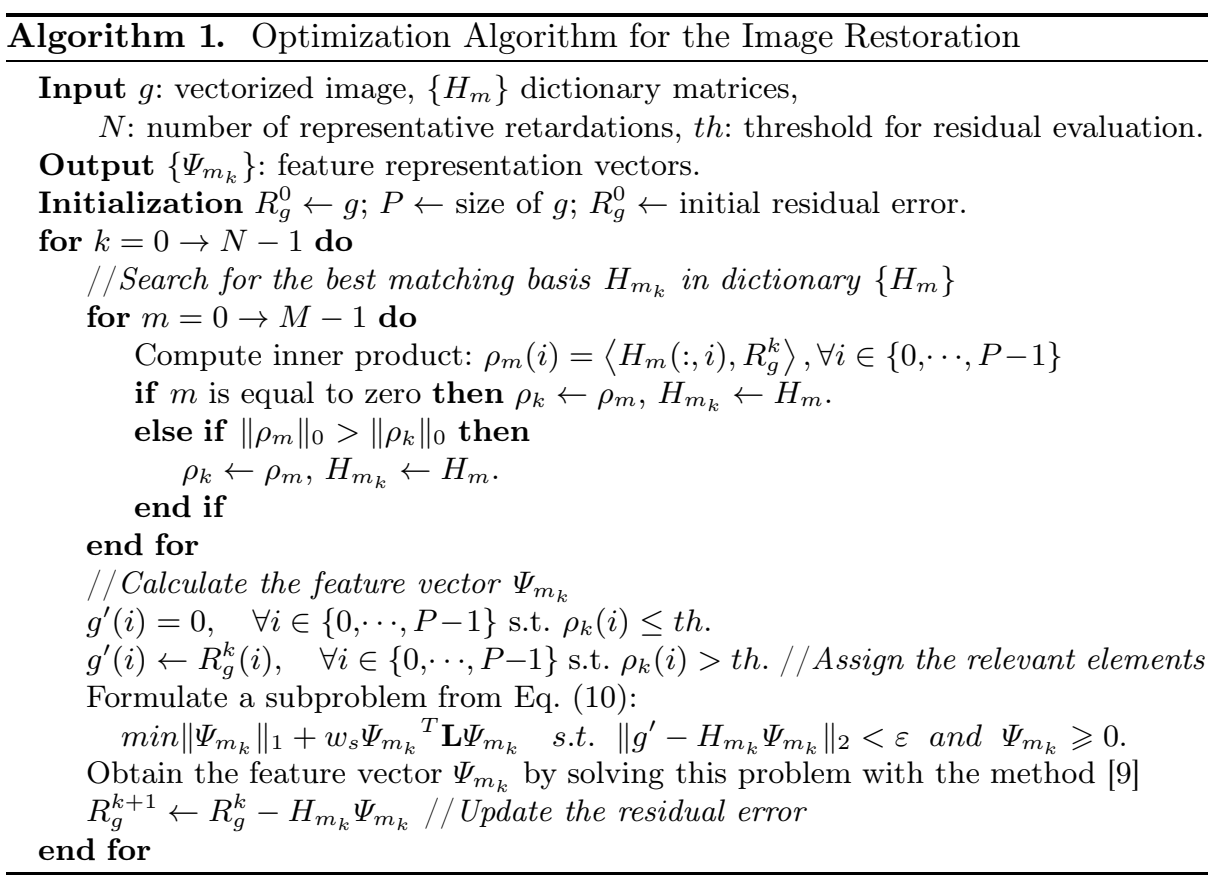

\section{Experiments and Discussions}

Data. The proposed approach was tested on three different sets of phase contrast images of $1040 \times 1392$ pixels. The specifications of the datasets are summarized in Table 1.

Table 1. Specifications of the Datasets

\begin{tabular}{|l|c|c|c|c|}
\hline & $\begin{array}{c}\text { Frame } \\
\text { number }\end{array}$ & Cell type & $\begin{array}{c}\text { Cell number } \\
\text { per image }\end{array}$ & Cell stages \\
\hline Seq1 & 500 & bovine aortic endothelial cell & 500 to $800+$ & intermitosis $/$ mitosis \\
\hline Seq2 & 600 & muscle stem of a progeroid & 50 to $300+$ & intermitosis $/$ mitosis \\
\hline Seq3 & 500 & C2C12 myoblastic stem cell & $300+$ & intermitosis/apoptosis(dead) \\
\hline
\end{tabular}

Parameters. Our algorithms involve three parameters: the dictionary size $M$, the number of representative phase retardations $N$, and number of classes for clustering $K$. We determined these parameters by investigating 10 frames for each sequence, which were uniformly sampled. For setting $M$, we plotted $M$ versus the average residual error in Eq. (3) and set $M$ to be 15 where the residual error levels off as shown in Fig. 1(a). We set $N$ to be 3 for dark cells, bright cells, and background. In addition to these three categories, we took into account two more categories, the boundary between either dark cells or bright cells and background, setting $k$ to be 5 , as shown in Figs. 1(b) and (c). 


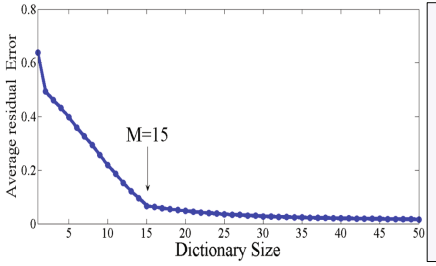

(a)

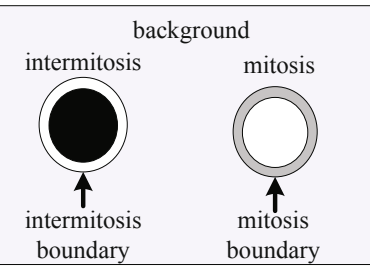

(b)

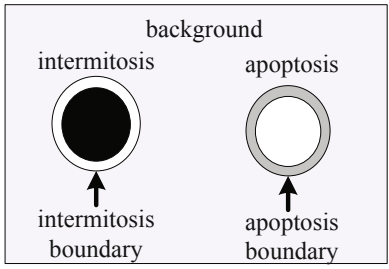

(c)

Fig. 1. (a) The average residual error decreases as $M$ increases and it levels off when $M$ is around 15. (b,c) Three representative retardations for intermitosis, mitosis/apoptosis(dead), and background; and five clustering classes for inner intermitosis, intermitosis boundary, inner mitosis/apoptosis, mitosis/apoptosis boundary, and background.
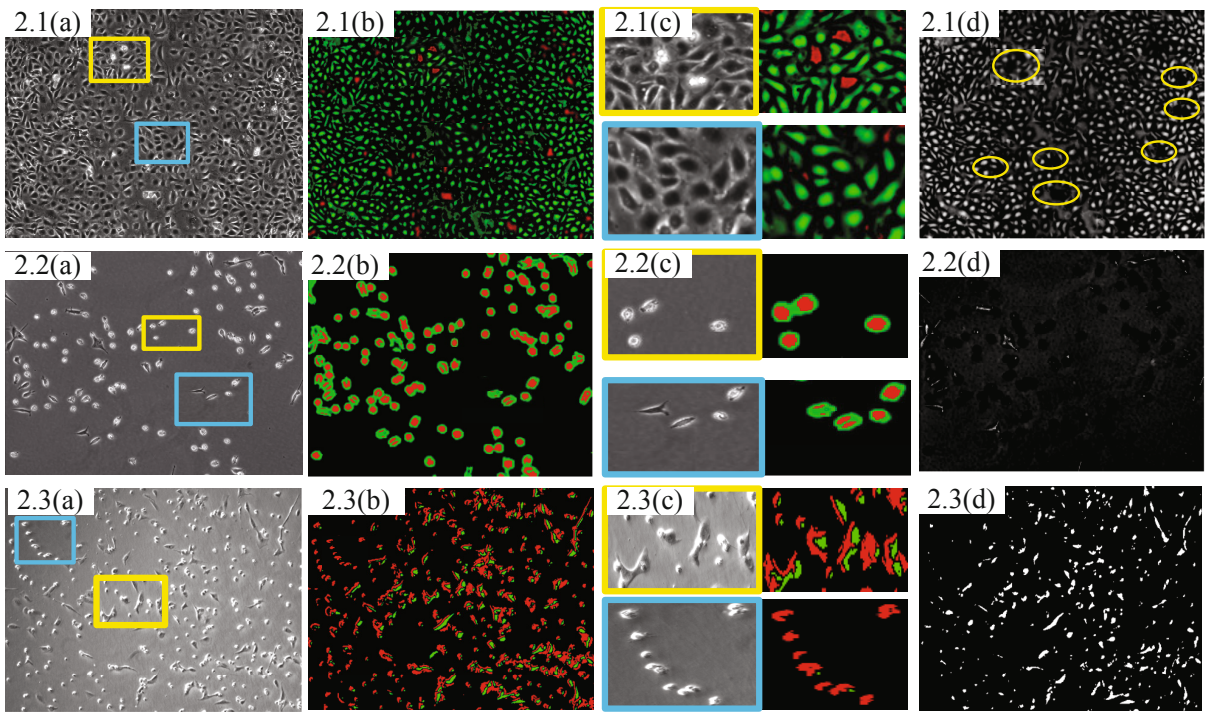

Fig. 2. The sample results of cell segmentation. Top, middle, and bottom rows show the results on Seq1, Seq2, and Seq3, respectively. (a) Original phase-contrast images, (b-c) segmentation results of the proposed method, (d) segmentation results of the previous method [7]. The intermitotic cells are marked with green color and the mitotic/apoptotic(dead) cells with red color. Yellow ellipses in Fig. 2.1(d) indicate missed mitotic cells. Figs. 2.2(d) and 2.3(d) also demonstrate that the previous method fails to detect most of mitotic/apoptotic(dead) cells.

Segmentation: Our dictionary-based approach achieved high quality segmentations as can be seen in Fig. 2. The method well detected bright cells, which undergo mitosis or apoptosis. On the other hand, the previous method [7] failed 
Table 2. Quantitative Results on Cell Segmentation

\begin{tabular}{|c|c|c|c|c|c|c|}
\hline & \multicolumn{2}{|c|}{ Seq.1 } & \multicolumn{2}{c|}{ Seq.2 } & \multicolumn{2}{c|}{ Seq.3 } \\
\hline & Proposed & Previous & Proposed & Previous & Proposed & Previous \\
\hline Average Precision & $93.1 \%$ & $87.8 \%$ & $95.3 \%$ & $34.8 \%$ & $94.5 \%$ & $31.4 \%$ \\
\hline Average Recall & $90.2 \%$ & $85.8 \%$ & $92.6 \%$ & $15.7 \%$ & $91.6 \%$ & $34.7 \%$ \\
\hline Average $F_{\text {score }}$ & 0.9154 & 0.8676 & 0.9412 & 0.1749 & 0.9302 & 0.3212 \\
\hline
\end{tabular}

Table 3. Quantitative Results on Cell Stage Classification

\begin{tabular}{|c|c|c|c|c|c|}
\hline \multicolumn{2}{|c|}{ Seq.1 } & \multicolumn{2}{c|}{ Seq.2 } & \multicolumn{2}{c|}{ Seq.3 } \\
\hline Precision & Recall & Precision & Recall & Precision & Recall \\
\hline $71.3 \%$ & $98.6 \%$ & $78.3 \%$ & $97.9 \%$ & $67.8 \%$ & $98.3 \%$ \\
\hline
\end{tabular}

to segment mitotic cells in Seq1 and Seq2 as well as apoptotic cells in Seq3, as shown in column (d) of Fig. 2. This result clearly demonstrates that the assumption of the previous method on the phase retardation being close to zero is not valid for bright cells.

Classification: The proposed method is able to not only segment cells, but also classify them among different stages. Fig. 2.1-2.(c) show zoom-in details on the classification between intermitotic cells (green) and mitotic/apoptotic(dead) cells (red). In Fig 2.3.(c), as cell death proceeds, more cells appear red on the results.

Evaluation: In order to evaluate our method quantitatively, we manually labeled every 50 th image in Seq1 (4125 annotated cells, $9.1 \times 10^{5}$ cell pixels), every 75th image in Seq2 (1459 annotated cells, $5.1 \times 10^{5}$ cells pixels), and every 100 th image in Seq3 (3915 annotated cells, $1.32 \times 10^{6}$ cell pixels). During the test, we skipped the frames used for training. We measured performance in terms of precision, recall, and $F$ score.

Table 2 demonstrates our method significantly outperforms the previous method [7]. The performance gap is more clear when data contains more mitotic or apoptotic(dead) cells. Our method adopts a combination of different phase retardations to detect various stages of cells.

Table 3 summarizes the performance of our algorithm on cell stage classification per frame. For this evaluation, we manually annotated 193 mitotic cells in Seq1, 396 mitotic cells in Seq2, and 596 apoptotic(or dead) cells in Seq3. The precision is not as high as recall since bright halos are often detected as bright cells. Mitosis and apoptosis are temporal events that occur over several frames; thus, the method that does not utilize temporal information and performs classification purely on per-frame features, like ours, obviously has limitation. However, the high recall indicates that our results can be used to provide a way to extract candidates for mitotic or apoptotic cells, for other methods that detect cellular events in a sequence (not per frame) by exploiting temporal contexts, e.g., [10]. 


\section{Conclusion}

In this paper, we propose a phase contrast image restoration method based on the dictionary representation of diffraction patterns. The dictionary corresponds to different phase retardations caused by specimens at different cell stages. We

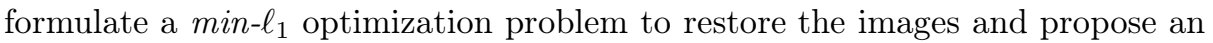
iterative algorithm to solve it. Experiments validate that our proposed method outperforms the previous method [7], particularly when cells undergo various stages. High quality restoration can benefit automated cell tracking and cell stage classification.

\section{References}

1. Murphy, D.: Phase Contrast Microscopy and Dark-Field Micorscopy. In: Fundamentals of Light Microscopy and Electronic Imaging, pp. 97-116. Wiley (2001)

2. Neumann, B., Held, M., Liebel, U., Erfle, H., Rogers, P., Pepperkok, R., Ellenberg, J.: High-throughput rnai screening by time-lapse imaging of live human cells. Nature Methods 3, 385-390 (2006)

3. Li, K., Miller, E.D., Chen, M., Kanade, T., Weiss, L.E., Campbell, P.G.: Cell population tracking and lineage construction with spatiotemporal context. Medical Image Analysis 12, 546-566 (2008)

4. Kachouie, N.N., Fieguth, P., Jervis, E.: Watershed deconvolution for cell segmentation. In: 30th Annual International Conference of the IEEE Engineering in Medicine and Biology Society, pp. 375-378 (2008)

5. Zimmer, C., Labruyere, E., Meas-Yedid, V., Guillen, N., Olivo-Marin, J.C.: Segmentation and tracking of migrating cells in videomicroscopy with parametric active contours: a tool for cell-based drug testing. IEEE Transactions on Medical Imaging 21, 1212-1221 (2002)

6. Padfield, D., Rittscher, J., Thomas, N., Roysam, B.: Spatio-temporal cell cycle phase analysis using level sets and fast marching methods. Medical Image Analysis 13, 143-155 (2009)

7. Yin, Z., Li, K., Kanade, T., Chen, M.: Understanding the Optics to Aid Microscopy Image Segmentation. In: Jiang, T., Navab, N., Pluim, J.P.W., Viergever, M.A. (eds.) MICCAI 2010, Part I. LNCS, vol. 6361, pp. 209-217. Springer, Heidelberg (2010)

8. Needell, D., Vershynin, R.: Signal recovery from incomplete and inaccurate measurements via regularized orthogonal matching pursuit. IEEE Journal of Selected Topics in Signal Processing 4, 310-316 (2010)

9. Sha, F., Lin, Y., Saul, L.K., Lee, D.D.: Multiplicative updates for nonnegative quadratic programming. Neural Comput. 19, 2004-2031 (2007)

10. Huh, S., Ker, D.F.E., Bise, R., Chen, M., Kanade, T.: Automated mitosis detection of stem cell populations in phasecontrast microscopy images. IEEE Trans. Med. Imaging 30, 586-596 (2011) 